\title{
Directional versus Omnidirectional Antennas for Energy Consumption and $k$-Connectivity of Networks of Sensors
}

\author{
Evangelos Kranakis * Danny Krizanc ${ }^{\dagger} \quad$ Eric Williams ${ }^{\ddagger}$
}

October 15, 2004

\begin{abstract}
A network is $k$-connected if it remains connected after the removal of any $k-1$ of its nodes. Assume that $n$ sensors, modeled here as (omni)directional antennas, are dropped randomly and independently with the uniform distribution on the interior of a unit length segment or a unit square. We derive sufficient conditions on the beam width of directional antennas so that the energy consumption required to maintain $k$-connectivity of the resulting network of sensors is lower when using directional than when using omnidirectional antennas. Our theoretical bounds are shown by experiment to be accurate under most circumstances. For the case of directional antennae, we provide simple algorithms for setting up a $k$-connected network requiring low energy.
\end{abstract}

\section{Introduction}

Communications networks are eliminating the barriers of distance and time by providing rapid access to information. New sensor systems currently under development add to these characteristics by providing the ability to function, autonomously, in unusually extreme and complex environments. They also have numerous applications in tele-medicine, transportation, tracking endangered species, detecting toxic agents, as well as monitoring the security of civil and engineering infrastructures.

Sensors are low power communication and sensing devices that can be embedded in the physical world (see Kahn et al [8], Sohrabi et al [21], Estrin et al [5]). Large scale sensor networks are formed by sensors that can be automatically configured after being dropped over a given region. It is expected that the cost of such devices will drop significantly in the near future (see [14], Agre et al [1], and Warneke et al [24]). Sensor nodes enable autonomy, self-configurability, and self-awareness, in the sense that they can assemble themselves automatically, adapt dynamically to failures, manage movement, and react to changes in network requirements. However, malfunctioning of individual sensors may well lead to operational failures resulting either in a disconnected network or failing to monitor a certain subregion.

* School of Computer Science, Carleton University, Ottawa, Ontario, K1S 5B6, Canada. Research supported in part by NSERC (Natural Sciences and Engineering Research Council of Canada) and MITACS (Mathematics of Information Technology and Complex Systems) grants.

${ }^{\dagger}$ Department of Mathematics and Computer Science, Wesleyan University, Middletown CT 06459, USA.

${ }^{\ddagger}$ Department of Mathematics and Computer Science, Wesleyan University, Middletown CT 06459, USA. 
Our paper addresses the problem of comparing the energy consumption between networks of omnidirectional and directional sensors under the assumption of maintaining network connectivity. Assume $n$ sensors are dropped randomly and independently with the uniform distribution over a region (which here we consider either a unit length segment or a unit square). A network is $k$-connected if it remains connected after the removal of any $k-1$ of its nodes. We investigate the impact of the size of the reachability radius of the sensors (given as a function of the total number $n$ of sensors and the number $k$ of faults) on the $k$-connectivity of the sensor system. We use this analysis to compare the energy consumption required for the $k$-connectivity of the resulting sensor network when using omnidirectional versus directional sensors. Our results show that significant savings are possible when directional antennae are used over omni-directional antennae, assuming the beam width of the directional antennae is sufficiently small. We compute theoretical bounds on the maximum beam width allowable in order to save energy and compare our theoretical results to experimentally derived bounds. As part of the derivation of our upper bound for directional antennae, we present simple algorithms for achieving $k$-connectivity in sensor networks.

\subsection{Model of sensors}

We consider two types of antennas: omnidirectional and directional. The former transmit their signal over a 360 degree angle and, for the purposes of this paper, any sensor within the reachability radius of this sensor will receive the signal. The latter are directional antennas that can be aimed and have a given beam width $\alpha$. They can be thought of as either being on a "swivel" that can be oriented towards a target or equivalently that each such sensor has multiple antennas each occupying a sector with beam width $\alpha$ so as to cover a 360 degree angle (in fact $\lceil 2 \pi / \alpha\rceil$ of these antennas would suffice). However, the sensor does not necessarily have to activate all these antennas at the same time. Instead, it will aim at a neighboring "target region or node" by activating the appropriate antenna so as to cover a region in a given direction.

\subsection{Energy consumption}

In any wireless network signals must be transmitted and received with sufficient strength in order to be properly detected and interpreted. For any kind of unguided, wireless media the signal disperses and falls off with distance over the transmission medium. Although attenuation is in general a complex function of the distance and the makeup of the atmosphere, a significant cause of signal degradation is simply free space loss which is due to the fact that the signal spreads over an ever larger area. For an ideal isotropic antenna free space loss is

measured as the ratio of the transmitted to received power and is equal to $\frac{(4 \pi d)^{2}}{\lambda^{2}}$, where $\lambda$ is the carrier wavelength, and $d$ is the propagation distance between antennas. In particular, the energy required by an antenna to reach all hosts within its radius is proportional to the area covered. Thus, with a reachability radius $r$ an omnidirectional antenna will consume power proportional to $\pi r^{2}$ (the area of a circle with radius $r$ ) while a directional antenna with beam width $\alpha$ radians will consume power proportional to $\frac{\alpha}{2} r^{2}$, whereby we assume that the signal is transmitted over the primary lobe and the power consumed by the remaining lobes is negligible. For additional information on antenna performance see Ramanathan [18] and on antenna theory see Balanis [2]. 


\subsection{Results of the paper}

The core of the paper is divided into two sections. First, in Section 2 we consider the case where the sensors are dropped on a unit length line segment. In Section 3 we consider the case where the sensors are dropped on a unit square. In both cases first we provide a sensor orientation algorithm and subsequently we study the energy consumption of the resulting sensor network. We also give a sufficient condition on the beam width of the antenna so that directional sensors consume less energy to achieve the same connectivity of the resulting sensor network. Table 1 summarizes our theoretical results. In Section 4 we provide experimental analysis.

\begin{tabular}{|l|l|}
\hline & Threshold beam width \\
\hline Unit Segment & $\frac{\pi}{2} \cdot\left(\frac{\ln n+k \ln \ln n+\ln (k !)-c}{\ln n+(2 k+1) \ln \ln n+c}\right)^{2}$ \\
\hline Unit Square & $\frac{2}{5(k+1)} \cdot\left(\frac{\ln n+k \ln \ln n+\ln (k !)-c}{\ln n+k \ln \ln n+c}\right)$ \\
\hline
\end{tabular}

Table 1: For the threshold value of the beam width indicated in the right column the energy consumption of a sensor network of $n$ directional sensors is below the energy consumption of a sensor network of $n$ omnidirectional sensors so as to achieve $(k+1)$-connectivity with probability at least $e^{-e^{-c}}-e^{-e^{c}}$.

\subsection{Related work and preliminaries}

Directional antennas have not been explored widely in the context of ad-hoc networks. Some recent papers exploring multiple beam antennas in order to increase throughput, and reduce delay and routing overhead include $[9,13,18,22]$. To date, however, we are not aware of any work that has considered a comparison of the energy efficiency of omnidirectional versus directional antennas with respect to connectivity properties of the network. Related to our work is the paper of Shakkottai et al [20] which addresses coverage and connectivity for a restricted model of omnidirectional sensors occupying the vertices of a unit square grid and to the paper of Kranakis et al [10] which investigates the more general model of directional sensors with given beam width occupying arbitrary positions (as opposed to grid points) in the interior of the unit square.

Useful for our analysis is the coupon collector's problem and its extensions. In particular, an extension of the coupon collectors problem is that of determining the threshold for the number (denoted by $X^{(k)}$ ) of selections (coupons) required in order to collect at least $k+1$ copies of each coupon type. It is well-known (see Motwani et al. [12][Exercise 3.11]) that the sharp threshold is centered at $n(\ln n+k \ln \ln n)$, i.e., for any integer $k \geq 0$ and constant $c$,

$$
\lim _{n \rightarrow \infty} \operatorname{Pr}\left[X^{(k)}>n(\ln n+k \ln \ln n+c)\right]=e^{-e^{-c}} .
$$

It is useful to note for $c>0$ large enough the term $e^{-e^{-c}}$ in the righthand side of Equation 1 is arbitrarily close to 1 and for $c<0$ large enough it is arbitrarily close to 0 .

Valuable for our theoretical analysis are also the studies on thresholds for the connectivity and minimum node degree, as well as general thresholds for monotone properties in geometric disk graphs that can be found in the work of Penrose $[15,16,17]$. Related bounds can be found in $[6,7,19,23]$ 


\section{Sensors on a Unit Length Line Segment}

In this section we limit our region to the unit length segment. We consider $(k+1)$-connectivity and contrast the energy consumption of omnidirectional versus directional antennas. For clarity of exposition, we separate the connectivity analysis for omnidirectional and directional antennas.

\subsection{Omnidirectional sensors}

Assume that $n$ omnidirectional sensors are dropped randomly and independently with the uniform distribution on the interior of a unit segment. For any integer $k \geq 1$ and real number constant $c$ let the sensors have identical radius $r$, given by the formula

$$
r=\frac{\ln n+k \ln \ln n+\ln (k !)-c}{n} .
$$

The main result of Penrose [15][Theorems 1.1 and 1.2] states that for the toroidal distance metric on a unit segment and the radius given by Identity 2

$$
\lim _{n \rightarrow \infty} \operatorname{Pr}[\text { network is }(k+1) \text {-connected }]=e^{-e^{c}} .
$$

The toroidal distance metric differs from the usual distance metric on a unit segment only in the wraparound boundary effects. Therefore Formula 3 gives an upper bound on the probability of achieving $(k+1)$-connectivity on a unit segment with the usual distance metric. Therefore we have the following theorem.

Theorem 1 Consider omnidirectional antennas, with reachability radius $r$ given by Formula 2, and suppose that $k \geq 0$ is an integer and $c>0$ is a real. Assume $n$ omnidirectional antennas are dropped randomly and independently with the uniform distribution on the interior of a unit segment. Then

$$
\lim _{n \rightarrow \infty} \operatorname{Pr}[\text { network is }(k+1) \text {-connected }] \leq e^{-e^{c}} .
$$

Thus, for the radius chosen by Formula 2 the network is $(k+1)$-connected with probability as indicated by Equation 4 .

\subsection{Directional sensors}

Consider the case of directional sensors each with a single antennae of beam width $\alpha$ that may be oriented in any direction. (We note that in the case of multiple antennae, an energy saving is trivially possible for any beam width by using two opposing antennae that cover the segment using the same radius as required by the omnidirectional case.) It is fairly easy to show that by aiming alternately $k+1$ antennae to the right along the segment followed by $k+1$ antennae to the left and insuring that each sensor reaches at least $2 k+2$ other sensors, the resulting network is $k$-connected. For each sensor, we choose the radius to be

$$
r=2 \cdot \frac{\ln n+(2 k+1) \ln \ln n+c}{n}
$$

and partition the unit interval into $\frac{2}{r}$ subintervals each of length $\frac{r}{2}$. We must aim their beam in such a way that $k$-connectivity of the resulting network is guaranteed. Using Equation 1, 


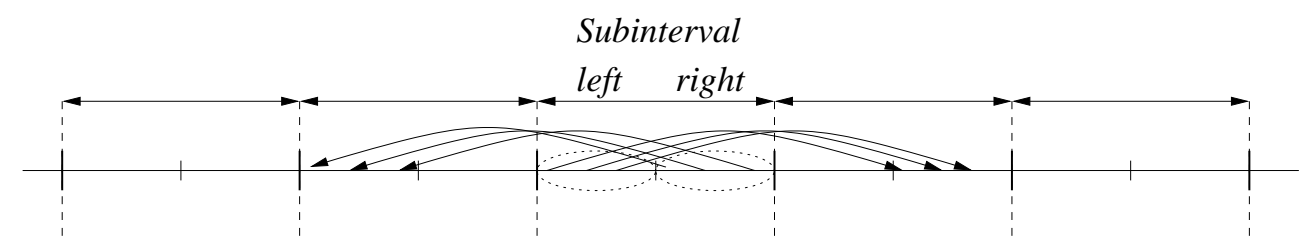

Figure 1: Alternating the beam direction of the sensors from one subinterval to the next.

it is easy to establish that each subinterval contains $2 k+2$ sensors with probability at least $e^{-e^{-c}}$. Next we divide the sensors in each subinterval into two (approximately) equal size parts: the leftmost half and the rightmost half (see Figure 1). For each subinterval we direct the leftmost half of the sensors (at least $k+1$ ) to the right and the rightmost half of the sensors (also at least $k+1$ ) to the left. We can prove the following theorem.

Theorem 2 Consider directional antennas with given beam width $\alpha$, reachability radius $r$ given by Formula 5, and suppose that $k \geq 0$ is an integer and $c>0$ is a real. Assume $n$ directional antennas are dropped randomly and independently with the uniform distribution on the interior of a unit length segment. Then

$$
\lim _{n \rightarrow \infty} \operatorname{Pr}[\text { network is }(k+1) \text {-connected }] \geq e^{-e^{-c}} .
$$

\subsection{Comparison of energy consumption}

Omnidirectional sensors transmit the signal over an angle $2 \pi$. In order to achieve $(k+1)$ connectivity the resulting energy consumption $\mathcal{E}_{\text {oMNI }}^{(k)}$ of the network satisfies

$$
\mathcal{E}_{\text {OMNI }}^{(k)} \geq n \cdot \pi \cdot\left(\frac{\ln n+k \ln \ln n+\ln (k !)-c}{n}\right)^{2},
$$

asymptotically in $n$ with probability at least $1-e^{-e^{c}}$. This can be contrasted with the energy consumption $\mathcal{E}_{\text {DIRE }}^{(k)}$ required to achieve $k+1$-connectivity of a network of directional sensors with beam width $\alpha$ (measured in radians). In particular, $\mathcal{E}_{\text {DIRE }}^{(k)}$ satisfies

$$
\mathcal{E}_{\mathrm{DIRE}}^{(k)} \leq n \cdot \frac{\alpha}{2} \cdot\left(2 \cdot \frac{\ln n+(2 k+1) \ln \ln n+c}{n}\right)^{2},
$$

asymptotically in $n$ with probability at least $e^{-e^{-c}}$. A simple calculation yields that asymptotically in $n$ if

$$
n \cdot \frac{\alpha}{2} \cdot\left(2 \cdot \frac{\ln n+(2 k+1) \ln \ln n+c}{n}\right)^{2} \leq n \cdot \pi \cdot\left(\frac{\ln n+k \ln \ln n+\ln (k !)-c}{n}\right)^{2}
$$

then $\mathcal{E}_{\text {DIRE }}^{(k)} \leq \mathcal{E}_{\text {OMNI }}^{(k)}$. We get the following result.

Theorem 3 Consider an experiment in which $n$ sensors are dropped randomly and independently in the interior of a unit length segment. Suppose that $k \geq 0$ is an integer and $c>0$ is a real. Then

$$
\alpha \leq 2 \pi \cdot\left(\frac{\ln n+k \ln \ln n+\ln (k !)-c}{\ln n+(2 k+1) \ln \ln n+c}\right)^{2}
$$


is a sufficient condition so that $\mathcal{E}_{\mathrm{DIRE}}^{(k)} \leq \mathcal{E}_{\mathrm{OMNI}}^{(k)}$ asymptotically in $n$ with probability at least $e^{-e^{-c}}-e^{-e^{c}}$.

\section{Sensors on a Unit Square}

In this section we limit our region to the unit square. We consider $(k+1)$-connectivity and contrast the energy consumption of omnidirectional versus directional antennas. We consider connectivity separately for omnidirectional and directional antennas.

\subsection{Omnidirectional sensors}

Assume that $n$ omnidirectional sensors are dropped randomly and independently with the uniform distribution on the interior of a unit square. For any integer $k \geq 0$ and real number constant $c$ let the sensors have identical radius $r$, given by the formula

$$
r=\sqrt{\frac{\ln n+k \ln \ln n+\ln (k !)-c}{n \pi}} .
$$

The main result of Penrose [15][Theorems 1.1 and 1.2] states that for the toroidal distance metric on a unit square and the radius given by Identity 8

$$
\lim _{n \rightarrow \infty} \operatorname{Pr}[\text { network is }(k+1) \text {-connected }]=e^{-e^{c}} .
$$

The toroidal distance metric differs from the usual distance metric on a unit square only in the wraparound boundary effects. Therefore Formula 9 gives an upper bound on the probability of achieving $(k+1)$-connectivity on a unit square with the usual distance metric. Therefore we have the following theorem.

Theorem 4 Consider omnidirectional antennas, with reachability radius $r$ given by Formula 8, and suppose that $k \geq 0$ is an integer and $c>0$ is a real. Assume $n$ omnidirectional antennas are dropped randomly and independently with the uniform distribution on the interior of a unit square. Then

$$
\lim _{n \rightarrow \infty} \operatorname{Pr}[\text { network is }(k+1) \text {-connected }] \leq e^{-e^{c}} .
$$

Thus, for the radius chosen by Formula 8 the network is $(k+1)$-connected with probability as indicated by Equation 10 .

\subsection{Directional sensors}

Consider the case of directional sensors with $k+1$ beams, where $k \geq 0$ is an integer. Fix $k$ and a constant $c>0$. Partition the unit square into $\frac{1}{r^{2}}$ subsquares or blocks each of side $r$, where

$$
r=\sqrt{\frac{\ln n+k \ln \ln n+c}{n}} .
$$

Let the reachability radius $r^{\prime}$ of the directional sensors be equal to the length of the diagonal of a rectangle with dimensions $r \times(2 r)$ (see Figure 2), i.e.,

$$
r^{\prime}=\sqrt{\frac{5(\ln n+k \ln \ln n+c)}{n}}=r \sqrt{5} .
$$


Let $N^{(k)}$ be the random variable that counts the number of sensors to be dropped so that each subsquare contains $k+1$ sensors. In view of Identity 1 ,

$$
\lim _{n \rightarrow \infty} \operatorname{Pr}\left[N^{(k)}>\frac{1}{r^{2}}\left(\ln \left(\frac{1}{r^{2}}\right)+k \ln \ln \left(\frac{1}{r^{2}}\right)+c\right)\right]=e^{-e^{-c}} .
$$

Now assume that $n$ sensors each of radius $r$ (given in Equation 11) are dropped on the interior of the unit square. Since $n>1 / r^{2}$, we have that

$$
n=\frac{n}{\ln n+k \ln \ln n+c} \cdot(\ln n+k \ln \ln n+c)>\frac{1}{r^{2}}\left(\ln \left(\frac{1}{r^{2}}\right)+k \ln \ln \left(\frac{1}{r^{2}}\right)+c\right) .
$$

By Equation 1 we have that each subsquare will have $k+1$ sensors with probability at least $e^{-e^{-c}}$. Now we must provide an "antenna orientation" algorithm to direct the sensor beams in such a way that connectivity in the unit square is guaranteed.

Number the sensors in a given square $1, \ldots, t+1$. (Assume sensors have unique identities and so they can order themselves. This set up phase can be done using broadcast in all directions.) For $i=1, \ldots, t+1$ the sensors numbered $i$ in each square form themselves into a hamiltonian cycle that visits every square using one of their $k+1$ antennae. Sensor $i$ in each square then uses its $k$ remaining antennae to point at sensor $j \neq i$ in its square. We claim the result is $k+1$-connected. Say sensor $i$ in block $B$ wants to talk to sensor $j$ in block $C$. If $k$ sensors fail, there is still a hamiltonian cycle (say nodes numbered $m$ ) that is completely alive. Node $i$ sends its message to node $m$ in block $B$, node $m$ in block $B$ sends the message to node $m$ in block $C$, which in turn sends it to node $j$ in block $C$. Therefore the network is $(k+1)$-connected. Two important points are the following

1. Some blocks have more than $k$ sensors. These sensors can be distributed arbitrarily among the cycles.

2. The longest any antennae has to reach is $r \sqrt{5}$, where $r$ is the side length required to have $k+1$ nodes per square. (Note: in the worst case an antennae must reach across the diagonal of a $2 r$ by $r$ rectangle.)

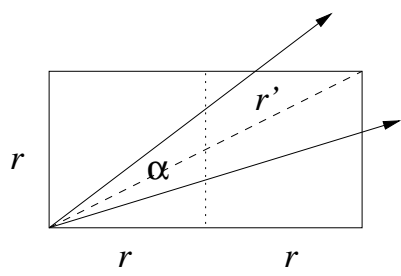

Figure 2: The radius $r^{\prime}$ of the directional sensors is determined by the geometry of two adjacent subsquares. in particular it must be chosen so that $r^{\prime} \geq r \sqrt{5}$.

In particular, regardless of the beam width, the resulting system of directional antennas must be $k+1$-connected with high probability. We can prove the following theorem.

Theorem 5 Consider directional antennas with given beam width $\alpha$, reachability radius $r$ given by Formula 12, and suppose that $k \geq 0$ is an integer and $c>0$ is a real. Assume $n$ 
directional antennas are dropped randomly and independently with the uniform distribution on the interior of a unit square. Then

$$
\lim _{n \rightarrow \infty} \operatorname{Pr}[\text { network is }(k+1) \text {-connected }] \geq e^{-e^{-c}} .
$$

We note that if we assume that the nodes have unique identities (e.g., if they can compute their geographic location through a GPS system) then the algorithm in Theorem 5 can be implemented fairly easily in a distributed manner. We further note that by using a small number of extra antennae aimed at sensors in the other adjacent subsquares can lead to an improvement in the diameter of the resulting sensor system from approximately $n / k$ to $\sqrt{n / k}$.

\subsection{Comparison of energy efficiency}

In this section we compare the energy consumption of a sensor network of $n$ omnidirectional versus $n$ directional sensors to attain $(k+1)$-connectivity. Let $\mathcal{E}_{\text {OMNI }}^{(k)}$ and $\mathcal{E}_{\text {DIRE }}^{(k)}$ be the energy consumption in the omnidirectional and directional case respectively, to attain $(k+1)$ connectivity.

Omnidirectional sensors transmit the signal over an angle $2 \pi$. In order to achieve $(k+1)$ connectivity $n$ omnidirectional sensors are necessary and the resulting energy consumption of the network satisfies

$$
\mathcal{E}_{\mathrm{OMNI}}^{(k)} \geq n \cdot \pi \cdot \frac{\ln n+k \ln \ln n+\ln (k !)-c}{n \pi},
$$

asymptotically in $n$ with probability at least $1-e^{-e^{c}}$. This can be contrasted with the energy consumption $\mathcal{E}_{\text {DIRE }}^{(k)}$ of a network of directional sensors with beam width $\alpha$ (measured in radians) which may transmit the signal over an angle $\alpha$. In this case, $\mathcal{E}_{\text {DIRE }}^{(k)}$ satisfies

$$
\mathcal{E}_{\mathrm{DIRE}}^{(k)} \leq n(k+1) \cdot \frac{\alpha}{2} \cdot \frac{5(\ln n+k \ln \ln n+c)}{n},
$$

asymptotically in $n$ with probability at least $e^{-e^{-c}}$. It is clear that, with high probability asymptotically in $n$ if

$$
\frac{5(k+1) \alpha}{2} \cdot(\ln n+k \ln \ln n+c) \leq \ln n+k \ln \ln n+\ln (k !)-c
$$

then $\mathcal{E}_{\text {DIRE }}^{(k)} \leq \mathcal{E}_{\text {OMNI }}^{(k)}$. A simple calculation yields the following theorem comparing the energy consumption of a sensor network of $n$ omnidirectional versus $n$ directional sensors to attain $(k+1)$-connectivity.

Theorem 6 Consider an experiment in which $n$ sensors are dropped randomly and independently in the interior of a unit square. Assume $k \geq 0$ is an integer and $c>0$ is a real. Then

$$
\alpha \leq \frac{2}{5(k+1)} \cdot\left(\frac{\ln n+k \ln \ln n+\ln (k !)-c}{\ln n+k \ln \ln n+c}\right)
$$

is a sufficient condition so that $\mathcal{E}_{\mathrm{DIRE}}^{(k)} \leq \mathcal{E}_{\mathrm{OMNI}}^{(k)}$ asymptotically in $n$ with probability at least $e^{-e^{-c}}-e^{-e^{c}}$. 


\section{Experimental Results}

The theoretical results presented above, while providing a true bound on $\alpha$, are based in part upon approximations and hold only asymptotically in $n$. In order to get an idea of how accurate our estimates are we provide the results of some simulations we performed on our simple model.

For both the one dimensional and two dimensional cases we considered values of $k$ ranging from 1 to 10 and values of $n$ ranging from 100 to 1000 by 100. Figure 3 depicts simulation results for unit segments. Figure 4 depicts simulation results for unit squares. Each experiment consisted of dropping $n$ sensors at random on either the unit segment or the unit square. For a given $k$, a lower bound on the radius required to achieve $k$-connectivity for omnidirectional antennae was obtained by finding the distance to the $k$-th nearest neighbor and an upper bound on the radius required to achieve $k$-connectivity using directional antennae was obtained using the algorithms described above. Each experiment was repeated 10,000 times and the average of all 10,000 runs along with error estimates were reported. The energy requirements in each case were computed using the model above and the ratios of the energy were plotted and compared to the theoretical result. We present the results of plotting $\alpha$ (the maximum beam width allowable to achieve energy savings) versus $n$ for $k=5$ as well as plotting $\alpha$ versus $k$ for $n=1000$. Plots for the other values of $k$ and $n$ are similar.

We observe that the theoretical bounds predict the shape of the curves quite well although for small values of $n$ and $k$ they significantly underestimate the value of $\alpha$ sufficient to ensure lower energy for the directional case. For the two dimensional case, we see that the theoretical curve approximates the experimental results quite well as $k$ increases. While the theoretical predictions should improve in accuracy as $n$ increases, it seems clear that a gap will always exist. We suspect that this is due to approximations made in our upper bounds for the directional case that may be improved using a better analysis.

\section{References}

[1] J. Agre and L. Clare, An integrated architecture for cooperative sensing networks, IEEE Computer, vol. 33, no. 5, May 2000, 106-108.

[2] C. A. Balanis, Antenna Theory: Analysis and Design, 2nd ed. New York: Wiley, 1997.

[3] D. Braginsky and D. Estrin, Rumor routing algorithm for sensor networks, 2001. Available at http://lecs.cs.ucla.edu/ estrin/.

[4] L. Doherty, L. E. Ghaoui, and K. S. J. Pister, Convex position estimation in wireless sensor networks, in Proceedings of IEEE Infocom, (Anchorage, AK), April 2001.

[5] D. Estrin, R. Govindan, J. Heidemann and S. Kumar: Next Century Challenges: Scalable Coordination in Sensor Networks. In Proc. 5th ACM/IEEE International Conference on Mobile Computing, MOBICOM'1999.

[6] A. Goel, S. Rai, B. Krishnamachari, Sharp Thresholds for Monotone Properties in Random Geometric Graphs. Stanford University, Manuscript, 2003. 

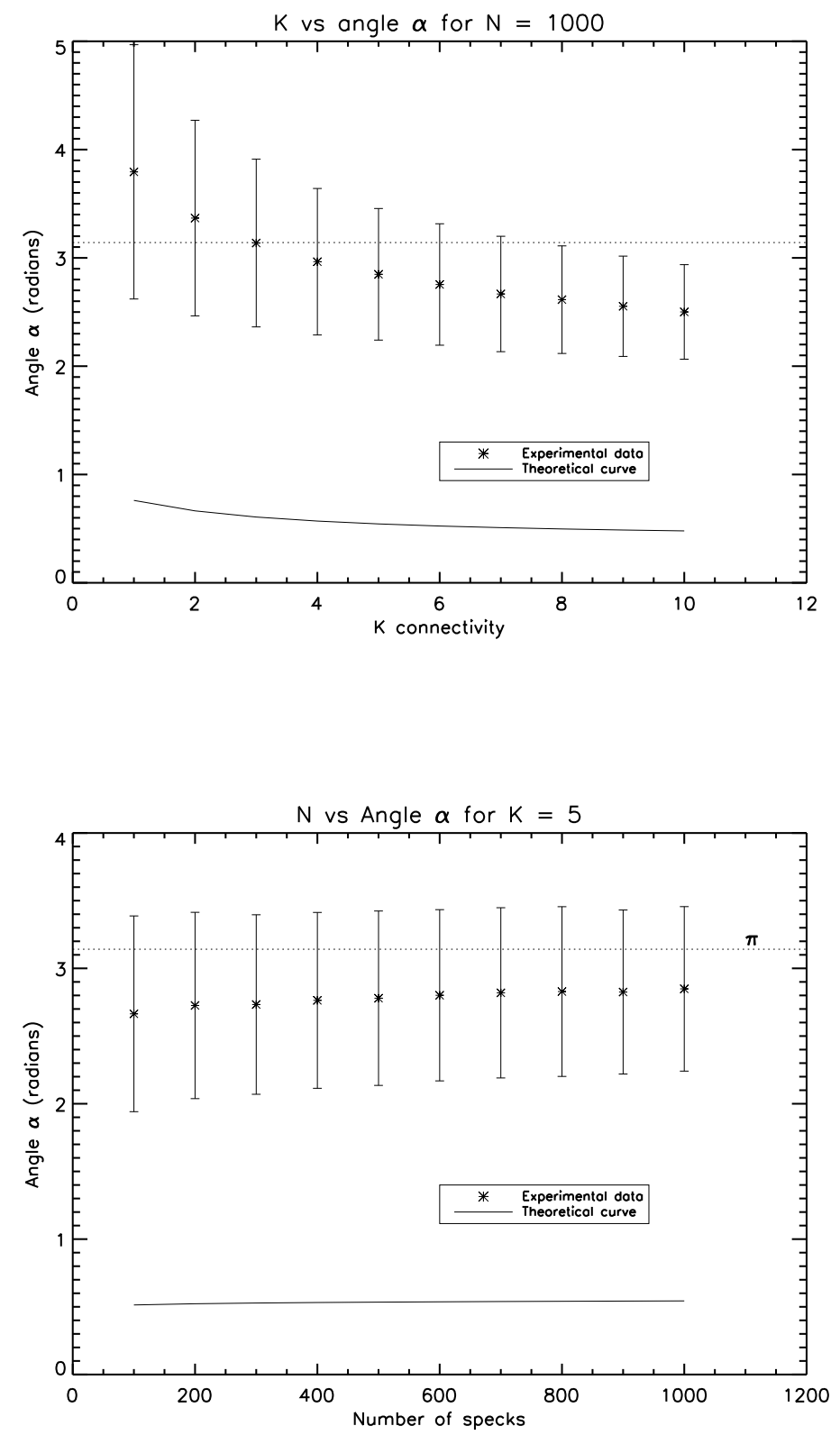

Figure 3: Simulation results for unit segment

[7] P. Gupta and P. R. Kumar, Critical Power for Asymptotic Connectivity in Wireless Networks. Stochastic Analysis, Control, Optimization, and Applications, Birkhauser, 1998.

[8] J. M. Kahn, R. H. Katz, and K. S. J. Pister, Mobile networking for smart dust, in 

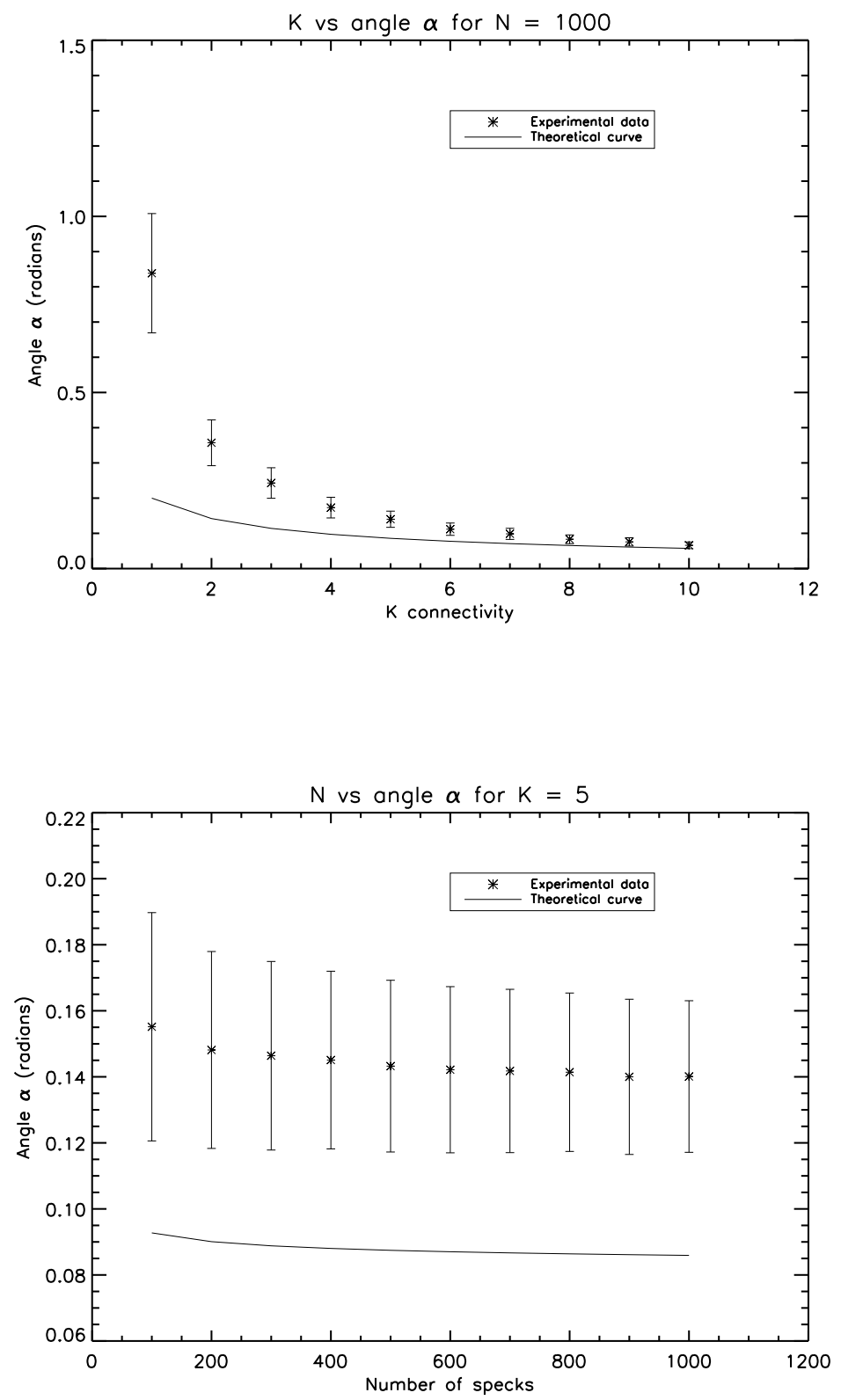

Figure 4: Simulation results for unit square

Proceedings of MobiCom 99, (Seattle, WA), August 1999.

[9] Y. B. Ko, V. Shankarkumar, and N. H. Vaidya, Medium access control protocols using directional antennas in ad-hoc networks, Proc. IEEE INFOCOM'2000, March 2000.

[10] E. Kranakis, D. Krizanc, J. Urrutia, Coverage and Connectivity in Networks with Direc- 
tional Sensors. In proceedings Euro-Par Conference, Pisa, Italy, August 31-September 3, 2004, Danelutto M., Vanneschi M., Laforenza D. (Eds.), Vol. 3149, Springer Verlag, LNCS.

[11] S. Meguerdichian, F. Koushanfar, M. Potkonjak, and M. Srivastava, Coverage problems in wireless ad-hoc sensor networks, in Proceedings of IEEE Infocom, (Anchorage, AK), 2001.

[12] R. Motwani, P. Raghavan, Randomized Algorithms, Cambridge University Press, 1995.

[13] A. Nasipuri, S. Ye, J. You, and R. E. Hiromoto, A MAC protocol for mobile ad hoc networks using directional antennas, Proc. IEEE Wireless Communications and Networking Conference (WCNC'2000), 2000.

[14] National Research Council, Embedded, Everywhere: A Research Agenda for Systems of Embedded Computers, Committee on Networked Systems of Embedded Computers, for the Computer Science and Telecommunications Board, Division on Engineering and Physical Sciences, Washington, DC, 2001.

[15] M. D. Penrose, On $k$-Connectivity for a Geometric Random Graph, Random Structures and Algorithms, 15, 145-164, 1999.

[16] M. D. Penrose, The Longest Edge of the Random Minimal Spanning Tree, The Annals of Applied Probability, 7(2) 1997, 340-361.

[17] M. D. Penrose, Random Geometric Graphs, Oxford University Press, 2003.

[18] R. Ramanathan, On the Performance of Ad Hoc Networks with Beamforming Antennas, In the Proceedings of ACM Symposium on Mobile Ad hoc Networking and Computing (MobiHoc'2001), 2001.

[19] P. Santi and D. Blough, The Critical Transmitting Range for Connectivity in Sparse Wireless Ad Hoc Networks, IEEE Transactions on Mobile Computing, to appear.

[20] S. Shakkottai, R. Srikant, N. Shroff, Unreliable Sensor Grids: Coverage, Connectivity and Diameter, In proceedings of IEEE INFOCOM, 2003, held in San Francisco, March 30 to April 2, 2003.

[21] K. Sohrabi, J. Gao, V. Ailawadhi, and G. Pottie, Protocols for self- organization of a wireless sensor network, IEEE Personal Communications, vol. 7, pp. 16-27, October 2000.

[22] A. Spyropoulos, and C.S. Raghavendra, Energy Efficient Communications in Ad Hoc Networks Using Directional Antennas, in proceedings of INFOCOM 2002, New York, June 23-27, 2002.

[23] P.-J. Wan and C.-W. Yi, Asymptotic Critical Transmission Radius and Critical Neighbor Number for k-connectivity in Wireless Ad Hoc Networks, Mobihoc, 2004, to appear.

[24] B. Warneke, M. Last, B. Leibowitz, and K. Pister, SmartDust: communicating with a cubic-millimeter computer, IEEE Computer, vol. 34, no. 1, January 2001, 44-51.

[25] W. Ye, J. Heidemann, and D. Estrin, An energy-efficient MAC protocol for wireless sensor networks, in Proceedings of IEEE Infocom, (New York, NY), June 2002. 